\title{
HÉRNIA DIAFRAGMÁTICA DIREITA TARDIA ASSOCIADA A HEPATOTÓRAX - RELATO DE CASO COM REVISÃO DA LITERATURA
}

\section{Hight diaphragmatic hernia with hepatothorax-case report and literature review}

\author{
José Marcos PARREIRA, Maurício CHIBATA, James SKINOVSKY, \\ Nestor SAUCEDO-JÚNIOR, Alfredo Mylonas MARTINS, Fernando Roberto SCHMIDT
}

ABCDDV/668

Parreira JM, Chibata M, Skinovsky J, Saucedo-Júnior N, Martins AM, Schimidt FR. Hérnia diafragmática direita tardia associada a hepatotórax - relato de caso com revisão da literatura. ABCD Arq Bras Cir Dig 2009;22(3):183-5

RESUMO - Introdução - Lesões diafragmáticas traumáticas ainda permanecem como um grande desafio diagnóstico na sala de emergência. Essas injúrias são entidades incomuns cujos diagnósticos passam despercebidos, resultando em hérnias diafragmáticas crônicas, podendo evoluir para estrangulamento. Relato do caso - Paciente com injúria diafragmática direita tardia associada a hepatotórax diagnosticada três anos após o trauma original. O comprometimento respiratório e dor abdominal em quadrante superior foram os principais sintomas que levaram à investigação diagnóstica. Ele foi manejado através de laparotomia e o defeito fechado mediante reparo primário, sem o uso de telas. Não houve complicações no pós-operatório, com alta após seis dias. Conclusão - As hérnias diafragmáticas tardias associadas a hepatotórax são raras. Estudos radiográficos e tomografias computadorizadas são os métodos de escolha para o correto diagnóstico. Tanto a toracotomia quanto a laparotomia podem ser realizadas para o reparo cirúrgico.

DESCRITORES - Hernia diafragmática.

\section{INTRODUÇÃO}

A hérnia diafragmática traumática é fenômeno pouco encontrado na prática clínica. Esse tipo de injúria ocorre de forma relativamente infrequente $(0,8 \%$ a $8,8 \%$ de todos os traumas) sendo, entretanto, o diagnóstico geralmente perdido durante a avaliação inicial na emergência ${ }^{18}$. Injúrias diafragmáticas não-reconhecidas podem ainda estar presentes em mais de $66 \%$ de pacientes com politrauma ${ }^{22}$. Em lesões contusas, esse tipo de ruptura acomete em $65 \%$ a $85 \%$ a hemicúpula diafragmática esquerda, $15 \%$ a $35 \%$ à direita, sendo $1 \%$ a $12 \%$ com comprometimento bilateral. Em relação às manifestações clínicas, elas dependem da extensão do órgão dentro do saco herniário, e das modificações funcionais e orgânicas sofridas se o órgão comprometido está fixo ou móvel. Acerca dos sintomas, aí estão incluídas queixas como dor escapular, distensão ou imobilidade do hemitórax, dispnéia, ruídos hidroaéreos audíveis no hemitórax afetado, congestão e edema hepático, hipertensão portal e eventualmente até síndrome de Budd-Chiari. $\mathrm{O}$ exame radiográfico é o melhor no screening; porém, apenas cerca de $50 \%$ dos pacientes apresentarão algum tipo de alteração. Embora a tomografia computadorizada axial seja relatada na literatura como exame capaz de elevar a sensibilidade diagnóstica para mais de $60 \%$, a helicoidal tem sido descrita como a técnica imaginológica mais sensível ${ }^{13}$. Outras modalidades diagnósticas ainda incluem o ultrassom,

Trabalho realizado no Departamento de Cirurgia Geral da Irmandade da Santa Casa de Misericórdia, Curitiba, Paraná, Brasil

Correspondência: Nestor Saucedo Jr, e-mail: nestor_sau@yahoo.com.br ressonância nuclear magnética, cintilografia de fígado e baço, laparoscopia e toracoscopia.

Para a correção, o reparo das hérnias é invariavelmente cirúrgico. Em situações agudas, a laparotomia é a primeira escolha como rota para acesso à lesão diafragmática. Em casos mais tardios, toracotomia ou laparotomia deve ser realizada, muito embora tanto a laparoscopia quanto a toracoscopia tenham sido relatadas na literatura como ferramentas de reparo de lesões diafragmáticas traumáticas ${ }^{10}$.

\section{RELATO DO CASO}

Mulher de 44 anos foi admitida com queixas de dispnéia e dor abdominal intermitente em quadrante superior direito do abdome. Na admissão relatava história pregressa de acidente automobilístico três anos antes resultando, na época, em fratura pélvica apenas. No exame físico, havia diminuição dos ruídos adventícios em hemitórax direito. Exames laboratoriais não possuíam alterações dignas de nota. $\mathrm{O}$ exame radiográfico de tórax solicitado demonstrou elevação da hemicúpula diafragmática direita (Figura 1). Tomografia computadorizada e ressonância nuclear magnética confirmaram o posicionamento do lobo hepático direito dentro da cavidade torácica à direita (Figura 2). Ela foi submetida à celiotomia longitudinal mediana. Na inspeção da cavidade, um defeito aproximado de $15 \mathrm{~cm}$ de diâmetro foi visualizado na hemicúpula diafragmática direita, com herniação de todo o lobo direito do fígado e vesícula biliar para o interior da cavidade pleural (Figura 3). Após o desbridamento das adesões pleurais, o reposicionamento do fígado foi realizado. $\mathrm{O}$ defeito foi reparado 


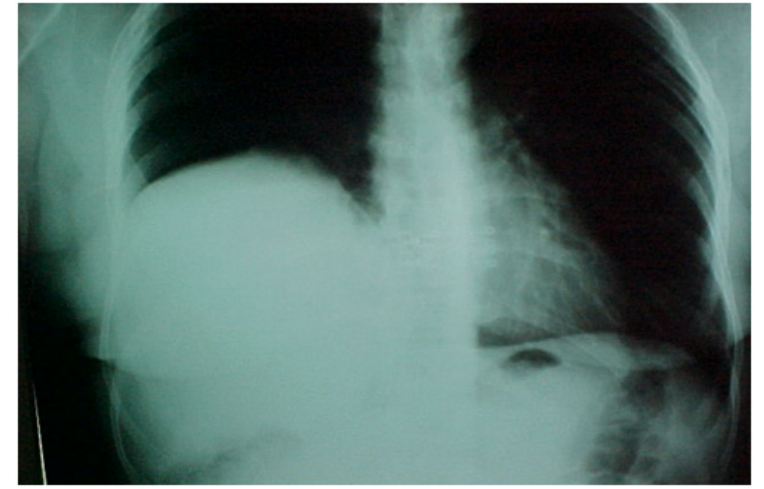

FIGURA 1 - Radiografia de tórax demonstrando elevação da hemicúpula diafragmática direita

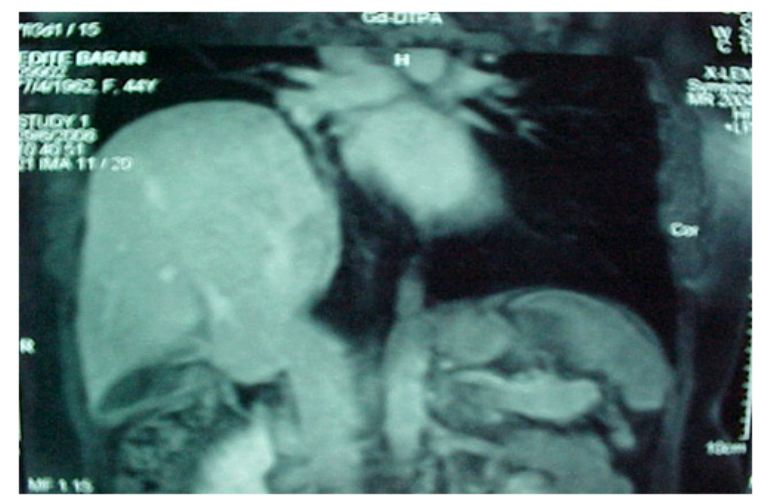

FIGURA 2 - Ressonância nuclear magnética demonstrando o deslocamento do fígado para dentro da cavidade torácica

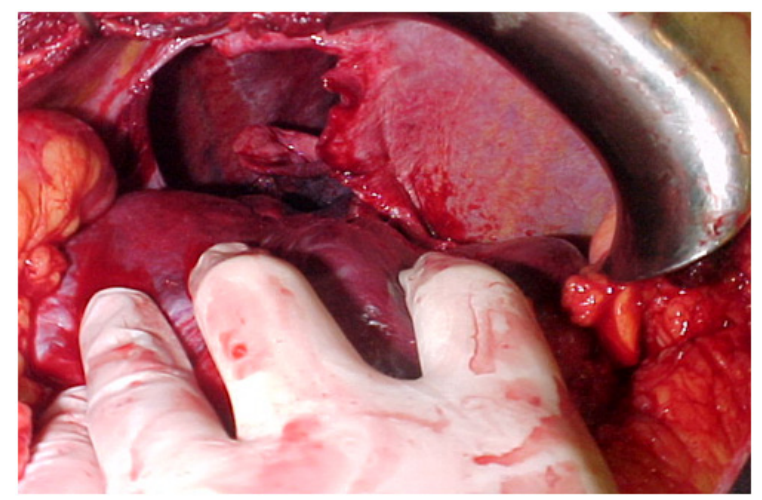

FIGURA 3 - Imagem intra-operatória monstrando o defeito diafragmático

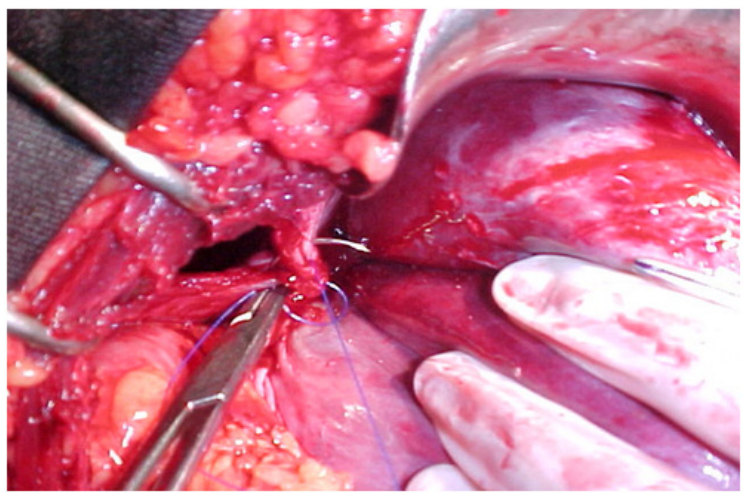

FIGURA 4 - Reparo primário do defeito diafragmático com fio de polipropileno com sutura direta, utilizando-se fio inabsorvível (Figura 4). O tórax à direita também foi drenado. Após seis dias de pósoperatório sem intercorrências, ela recebeu alta. Um ano após permanecia assintomática.

\section{DISCUSSÃO}

As hérnias diafragmáticas podem ser tanto congênitas quanto adquiridas. Em relação às congênitas, há três tipos - póstero-lateral, subcostoesternal e as hérnias esofagianas. Já, as hérnias adquiridas são geralmente resultado de trauma penetrante tóraco-abdominal, contuso ou ainda iatrogêni$\mathrm{co}^{2}$. Esse tipo de lesão tende a ocorrer em aproximadamente $3 \%$ a $9 \%$ de todos os traumas torácicos ou abdominais. Para traumas contusos severos tóraco-abdominais, a incidência é ainda mais elevada, atingindo patamares de até $22 \%$ em algumas séries. Moore et al. ${ }^{11}$ relatou para pacientes com lesões por armas brancas e feridas por armas de fogo porcentagem respectiva de $15 \%$ e $46 \%$ para injúrias diafragmáticas, enquanto que $4,5 \%$ dos traumas múltiplos contusos se apresentariam com lesões no diafragma ${ }^{1,11}$. Ainda, os traumas penetrantes representariam mais de $90 \%$ das causas de lesões diafragmáticas. Shah et al. ${ }^{20}$ and Sharma et al. ${ }^{21}$ relataram que o trauma contuso é responsável por $68 \%$ a $75 \%$ das injúrias e os penetrantes apenas $25 \%$ a $32 \%{ }^{20,21}$. Outro estudo demonstrou que o diafragma é lacerado em $10 \%$ a $15 \%$ das injúrias torácicas penetrantes e em $0,8 \%$ a $1,6 \%$ dos pacientes com traumas contusos.

As lesões diafragmáticas podem ser diagnosticadas imediatamente, em meses ou anos após o trauma. Lesões diafragmáticas despercebidas no trauma inicial têm sido relatadas entre $9,5 \%$ a $61 \%$ dos $\operatorname{casos}^{1}$. Ainda, casos de até 50 anos após o trauma já foram relatados na literatura ${ }^{10}$. Em relação às rupturas, estas tendem a ocorrer na região tendínea central ou na área de junção entre a parte tendínea e a muscular do diafragma ${ }^{22}$. Os defeitos tendem a ser largos, usualmente entre $10 \mathrm{a} 15 \mathrm{~cm}^{17}$. Os órgãos que podem herniar através do defeito incluem o estômago, cólon, omento, alças de delgado e fígado, nesta ordem de frequência ${ }^{14}$. A hemicúpula diafragmática direita geralmente é envolvida em $15 \%$ a $24 \%$ dos casos, a esquerda em $70 \%$ a $80 \%$, sendo que em $5 \%$ a $8 \%$ o comprometimento é bilateral. Esta diferença pode ser explicada pelo efeito biodinâmico do trauma contuso na região tóraco-abdominal, bem como a relação anatômica dos órgãos na cavidade abdominal.

Os sintomas e achados das hérnias diafragmáticas incluem diminuição do murmúrio vesicular $(64,2 \%)$, dispnéia (42,8\%), ruídos hidro-aéreos auscultáveis no tórax (35,7\%), náuseas e vômitos $(35,7 \%)$ e dor abdominal $(28,5 \%)^{16}$. As lesões agudas são frequentemente associadas à dispnéia, ortopnéia, dor torácica ou escapular, muitas vezes mimetizando um quadro de pneumotórax hipertensivo ${ }^{22}$. Estas lesões são também associadas a $40 \%$ das fraturas pélvicas, $25 \%$ dos casos de lesões hepáticas e esplênicas e uma incidência de 5\% nos casos de lesões de aorta torácica.

Inúmeras modalidades diagnósticas podem ser utilizadas na tentativa de se identificar a injúria diafragmática. A radiografia de tórax é geralmente o estudo de imagem solic- 
itado inicialmente. Esse tipo de exame apresenta sensibilidade de $46 \%$ para as rupturas à esquerda e $17 \%$ das rupturas à direita ${ }^{5}$. Alguns achados sugestivos incluem elevação do diafragma e deslocamento contralateral do mediastino ${ }^{9}$. A herniação da víscera abdominal pode ser evidente na radiografia de tórax quando o ar encontra-se contido em vísceras ocas, como o estômago, intestino delgado e cólon ${ }^{6}$. A tomografia computadorizada aumenta a sensibilidade entre $61 \%$ e $90 \%{ }^{3}$. Já a helicoidal apresenta-se como ferramenta diagnóstica de maior valor, com sensibilidade de $71 \%$ e especificidade de $100 \%^{8}$. A ressonância nuclear magnética, a ultrassonografia, os estudos contrastados do trato gastro-intestinal, a laparoscopia ou toracoscopia são também descritos como excelentes ferramentas diagnósticas no auxílio ao diagnóstico das lesões diafragmáticas em $70 \%$ a $85 \%$ dos $\operatorname{casos}^{12}$. As rupturas à direita têm sido diagnosticadas com sucesso mediante uso da ressonância nuclear magnética e ultrassom ${ }^{4}$. A cintilografia do fígado e baço com 99 mTc também possui seu espaço em casos de suspeita de lesão diafragmática ${ }^{7}$.

A melhor abordagem cirúrgica nas lesões agudas é, em último caso, determinado pela presença de alguma lesão associada ${ }^{6}$. Se o diagnóstico é tardio e não há preocupações em relação a lesões de vísceras, o reparo será provavelmente melhor corrigido via transtorácica ${ }^{2}$. Peker et al..$^{15}$ descreveu ainda reparos com sucesso de hérnias diafragmáticas tardias à direita através de laparotomia ${ }^{15}$. Com relação ainda ao tratamento, as injúrias devem ser sempre reparadas com fios não-absorvíveis. Os enxertos autólogos, homólogos ou sintéticos geralmente não são necessários ${ }^{23}$. Porém, quando o reparo primário é impossível, o defeito diafragmático pode ser corrigido com material prostético ${ }^{19}$. Além disso, na última década, inúmeros relatos de casos e estudos de casos-controle vêm descrevendo excelentes resultados de reparo das lesões diafragmáticas agudas e crônicas utilizando laparoscopia ${ }^{10}$. Ainda pela laparoscopia, tanto o reparo primário com fios inabsorvíveis quanto a utilização de telas sintéticas podem ser realizados de forma segura e com excelente reprodutibilidade ${ }^{10}$.

\section{CONCLUSÃO}

Este caso ilustra um raro caso de hérnia diafragmática direita corrigida com sutura primária. Nenhuma complicação foi relatada após seguimento de 12 meses.

Parreira JM, Chibata M, Skinovsky J, Saucedo-Júnior N, Martins AM, Schimidt FR. Hight diaphragmatic hernia with hepatothorax - case report and literature review. ABCD Arq Bras Cir Dig 2009;22(3):183-5

ABSTRACT - Introduction - Traumatic diaphragm lesions still remain problematic in diagnosis and handling. Case report - Patient with diaphragmatic hernia had the diagnosis made three years after car accident. At admission, he was with abdominal pain and respiratory distress. Submitted to image investigation, had the diagnosis done of a diaphragm herniation. Laparotomy revealed liver and adjacent viscera into the thorax and a diaphragm defect of $15 \mathrm{~cm}$. The defect was closed without the use any mash. There was no complications and in an outclinic assistance, one year later, he was asymptomatic. Conclusion - Diaphragmatic herniations are rare entities. Image examination is indicated and can do correct diagnosis in all cases. Laparotomy or thoracotomy must be use in defect reparation.

HEADINGS - Hernia, Diaphragmatic.

\section{REFERÊNCIAS}

1. Alimoglu O, Eryilmaz R, Sahin M, Ozsoy MS. Delayed traumatic diaphragmatic hernias presenting with strangulation. Hernia. 2004 Dec;8(4):393-6.

2. Armstrong PA, Miller SF, Brown GR. Diaphragmatic hernia seen as a late complication of laparoscopic cholecystectomy. Surg Endosc. 1999 Aug;13(8):817-8

3. Bergin D, Ennis R, Keogh C, Fenlon HM, Murray JG. The "dependent viscera" sign in CT diagnosis of blunt traumatic diaphragmatic rupture. AJR Am J Roentgenol. 2001 Nov;177(5):1137-40.

4. Dosios T, Papachristos IC, Chrysicopoulos H. Magnetic resonance imaging of blunt traumatic rupture of the right hemidiaphragm. Eur J Cardiothorac Surg. 1993;7(10):553-4.

5. Gelman R, Mirvis SE, Gens D. Diaphragmatic rupture due to blunt trauma: sensitivity of plain chest radiographs. AJR Am J Roentgenol. 1991 Jan;156(1):51-7.

6. Healy DG, Veerasingam D, Luke D, Wood AE. Delayed discovery of diaphragmatic injury after blunt trauma: report of three cases. Surg Today. 2005;35(5):407-10.

7. Hoang AD, De Backer D, Bouazza F, Capello M, De Francquen P, Rocmans P. Undiagnosed rupture of right hemidiaphragm--hepatothorax: a case report. Acta Chir Belg. 2002 Oct;102(5):353-5.

8. Killeen KL, Mirvis SE, Shanmuganathan K. Helical CT of diaphragmatic rupture caused by blunt trauma. AJR Am J Roentgenol. 1999 Dec;173(6):1611-6.

9. Koroglu M, Ernst RD, Oto A, Mileski WJ. Traumatic diaphragmatic rupture: can oral contrast increase CT detectability? Emerg Radiol. 2004 Jul;10(6):334-6.

10. Matthews BD, Bui H, Harold KL, Kercher KW, Adrales G, Park A, Sing RF, Heniford BT. Laparoscopic repair of traumatic diaphragmatic injuries. Surg Endosc. $2003 \mathrm{Feb} ; 17(2): 254-8$

11. Moore JB, Moore EE, Thompson JS. Abdominal injuries associated with penetrating trauma in the lower chest. Am J Surg. 1980 Dec;140(6):724-30.

12. Nau T, Seitz H, Mousavi M, Vecsei V. The diagnostic dilemma of traumatic rupture of the diaphragm. Surg Endosc. 2001 Sep;15(9):992-6.
13. Nchimi A, Szapiro D, Ghaye B, Willems V, Khamis J, Haquet L, Noukoua C, Dondelinger RF. Helical CT of blunt diaphragmatic rupture. AJRAm J Roentgenol. $2005 \mathrm{Jan} ; 184(1): 24-30$.

14. Nursal TZ, Ugurlu M, Kologlu M, Hamaloglu E. Traumatic diaphragmatic hernias: a report of 26 cases. Hernia. 2001 Mar;5(1):25-9.

15. Peker Y, Tatar F, Kahya MC, Cin N, Derici H, Reyhan E. Dislocation of three segments of the liver due to hernia of the right diaphragm: report of a case and review of the literature. Hernia. $2007 \mathrm{Feb}$;11(1):63-5.

16. Reina A, Vidaña E, Soriano P, Orte A, Ferrer M, Herrera E, Lorenzo M, Torres J, Belda R. Traumatic intrapericardial diaphragmatic hernia: case report and literature review. Injury. 2001 Mar;32(2):153-6.

17. Rodriguez-Morales G, Rodriguez A, Shatney CH. Acute rupture of the diaphragm in blunt trauma: analysis of 60 patients. J Trauma. 1986 May;26(5):438-44.

18. Rubikas R. Diaphragmatic injuries. Eur J Cardiothorac Surg. 2001 Jul;20(1):53-7.

19. Saito Y, Yamakawa Y, Niwa H, Kiriyama M, Fukai I, Kondo S, Fujii Y. Left diaphragmatic hernia complicated by perforation of an intrathoracic gastric ulcer into the aorta: report of a case. Surg Today. 2000;30(1):63-5.

20. Shah R, Sabanathan S, Mearns AJ, ChoudhuryAK. Traumatic rupture of diaphragm. Ann Thorac Surg. 1995 Nov;60(5):1444-9.

21. Sharma OP. Traumatic diaphragmatic rupture: not an uncommon entity--personal experience with collective review of the 1980's. J Trauma. 1989 May;29(5):678-82

22. Thoman DS, Hui T, Phillips EH. Laparoscopic diaphragmatic hernia repair. Surg Endosc. 2002 Sep;16(9):1345-9.

23. Wirbel RJ, Mutschler W. Blunt rupture of the right hemi-diaphragm with complete dislocation of the right hepatic lobe: report of a case. Surg Today. 1998;28(8):850-2.

Fonte de financiamento: não há Conflito de interesse: não há Recebido para publicação: 29/11/2008 Aceito para publicação: 20/01/2009 